\title{
Maintenance of the plant genome under natural light
} Anne Britt* and Kevin Culligan

\author{
Address: Section of Plant Biology, University of California, One Shields Ave. Davis, CA 95616, USA \\ Email: Anne Britt* - abbritt@ucdavis.edu \\ * Corresponding author
}

from Cell Biology of Nitric Oxide and Cell Death in Plants

Yalta, Ukraine, 8-II September 2004

Published: 3I May 2005

BMC Plant Biology 2005, 5(SuppI I):S7 doi:I0.I I86/I47I-2229-5-SI-S7

Solar UV interacts with and alters a number of cellular components. Among these, DNA is perhaps the most critical and irreplaceable target. The majority of UV-induced damage takes the form of pyrimidine dimers. These lesions act both as blocks to the progression of both replicative DNA polymerases and the RNA polymerase holoenzyme. Expression of "dimer bypass" DNA polymerases may also lead to permanent changes (mutations) in DNA sequence, by inserting potentially incorrect bases opposite the lesion. Thus the excision or reversal of UV-induced damage is important both in the soma, as it is required for the maintenance of gene expression, and in the germline, where error-free repair pathways maintain genomic integrity. The basic mechanisms of photoreactivation and excision repair of UV-induced dimers are well understood in plants, although we would like to learn more about the tissue-specificity and environmental regulation of these important UV-protective mechanisms. Field experiments suggest that repair of UV-induced dimers is not essential to the survival of Arabidopsis plants, nor is the expression of the sinapic acid esters that act as natural sunscreens. However, plants that are defective in both repair and sunscreen production die within hours of exposure to natural light.

Progression through the cell cycle in the presence of unrepaired DNA damage products leads to a progressive deterioration of the genome. During $\mathrm{S}$ phase, persisting lesions are either miscopied or produce daughter strand gaps opposite dimers, which are difficult to repair in an error-free manner. For this reason, cells respond to persisting DNA damage by arresting the cell cycle in order to provide time prior to the initiation of $S$ phase (G1/S arrest), the continuation of $S$ phase (intra-S arrest) or progression into $M$ (G2/M arrest). These DNA damage-induced cell cycle "checkpoints" are now being characterized in plants.
Given the homologies between plant and mammalian damage checkpoint genes, it is likely that many aspects of cell cycle regulation by UV-induced damage are shared between plants and animals.

One UV-induced mammalian response to persisting DNA damage is the induction of programmed cell death. This actively induced apoptosis and necrosis of damaged cells leads to the inflammation observed in sunburn. The induction of cell death has two beneficial effects: the resulting bursts of radicals are thought to further stimulate the repair response of neighboring cells, and the suicide of damaged cells precludes their possible progression into cancer. Plant cells can also be killed by very high doses of UV light, in what might be a programmed response (as the genomic "laddering" characteristic of programmed cell death occurs), but it is not clear that such a response occurs under natural light. Given plants natural resistance to the lethal effects of cancer, it is possible that plants differ from mammals in this aspect of UV-response, and lack a sensitive apoptotic response to DNA damage. 